\title{
Lack of association between the occurrence of Crohn's disease and occupational exposure to dairy and beef cattle herds infected with Mycobacterium avium subspecies paratuberculosis
}

\author{
D. A. Qual, ${ }^{*}$ J. B. Kaneene,† T. J. Varty, $¥$ R. Miller,† and C. O. Thoen*1 \\ *Department of Veterinary Microbiology and Preventive Medicine, College of Veterinary Medicine, lowa State University, Ames 50014 \\ †Center for Comparative Epidemiology, College of Veterinary Medicine, Michigan State University, East Lansing 48824 \\ ‡USDA/APHIS/VS, Madison, WI 53711
}

\begin{abstract}
A cross-sectional survey was conducted to identify associations between Crohn's disease (CD) and Mycobacterium avium ssp. paratuberculosis (Map) exposure. A questionnaire was used to collect information on exposure to cattle infected with Map, and personal and family history of CD in dairy and beef cattle producers with and without $M a p$-infected herds, and in veterinarians who did or did not have contact with Map-infected herds. Cases of CD were selected from respondents and matched 1:4 with controls on occupation, age, and sex. Multivariable conditional logistic regression was used to assess associations between Map exposure and CD. There were 3 cases of CD in 702 producers and 4 cases in 774 veterinarians, yielding a prevalence of $0.47 \%$. No association was found between exposure to JD and CD in any phase of the analysis. However, the number of cases of $\mathrm{CD}$ is not large and limits the power to detect important differences.
\end{abstract}

Key words: Johne's disease, Crohn's disease

\section{INTRODUCTION}

Crohn's disease (CD), a chronic inflammatory disease of the human intestine, shares some characteristics with Johne's disease (JD) in cattle, a chronic inflammatory disease of the bovine intestine caused by infection with Mycobacterium avium ssp. paratuberculosis (Map). Given the similarity of the 2 diseases and evidence of Map in CD patients (McFadden et al., 1987; Chiodini, 1989), considerable work has been conducted to draw causal associations between Map infection and CD in human patients, but different studies have yielded different results. Findings from one review (Behr and Schurr, 2006) indicated that the methods of most studies into causal associations between Map and CD lack sufficient

Received May 1, 2009.

Accepted March 9, 2010.

${ }^{1}$ Corresponding author: cthoen@iastate.edu standardization to generate reproducible prevalence estimates and strengths of association, which further fuels the ongoing controversy.

Many studies have found evidence for Map in CD patients (Lisby et al., 1994; Bull et al., 2003; Naser et al., 2004), but other studies have found no such evidence of Map (Van Kruiningen, 1999; Freeman and Noble, 2005; Feller et al., 2007), and some studies have found Map in patients that do not have CD (Bull et al., 2003; Naser et al., 2004). Findings that a combination of genetic mutations (CARD15, formerly NOD2) and Map infection are associated with the development of CD suggest that Map alone may not be sufficient to cause infection, but increased susceptibility due to mutation is needed to cause infection (Behr and Schurr, 2006). Because Map has been detected in pasteurized milk in the United States and the United Kingdom (Ellingson et al., 2005), any causal associations between Map and CD may represent a considerable public health concern, which will have a significant negative economic impact on the nation's cattle industry.

Ecological studies have been undertaken to determine whether associations exist between Map and CD, given the conflicting evidence of studies examining the presence or absence of Map in human CD patients. One study found spatial associations between areas of higher CD downwind of the Taff River in Wales, where bovine strains of Map have been cultured (Pickup et al., 2005), but other studies of exposure to Map through contact with cattle affected by JD found no associations between Map or JD and CD in the United Kingdom (Jones et al., 2006; Abubakar et al., 2007). To further explore the hypothesis that exposure to Map, through exposure to Map-infected cattle or dairy products, increases risk for CD in the United States, a cross-sectional study was conducted to 1) obtain information on the occurrence of Crohn's disease in dairy and beef cattle producers, their families, and veterinarians in Iowa, Michigan, and Wisconsin, and 2) determine if there were any associations between exposure to cattle infected with Map and the occurrence of $\mathrm{CD}$ in these populations. 


\section{MATERIALS AND METHODS}

\section{Study Population}

Study participants were selected based on their possible exposure to Map through contact with cattle, and consisted of 774 veterinarians and 702 dairy and beef producers from Michigan, Wisconsin, and Iowa. In Michigan, lists of potential participants were randomly selected from a list of veterinary practitioners provided by the Michigan Veterinary Medical Association, and from producer list frames created by the Michigan Agricultural Statistical Service. Dairy and beef producers and veterinarians in Wisconsin and Iowa were recruited at producer meetings and professional conferences and meetings.

\section{Case and Exposure Definitions}

Cases of $\mathrm{CD}$ in producers or veterinarians were defined as physician-confirmed diagnoses of the disease, as reported by the study respondent. Cattle producers and veterinarians were classified as being either exposed or unexposed to Map, based on their reported contact with cattle diagnosed as having JD, confirmed by fecal culture or typical histopathologic lesions, and supported by positive serology, PCR, or both, for Map.

\section{Data Collection}

Data were collected via pretested, self-administered questionnaires that were mailed (Michigan) or distributed at conferences and meetings (Wisconsin and Iowa) in 2000. Second and third mailings to nonrespondents in Michigan were conducted to improve response rates. The questionnaires collected information about respondent (age and sex), diagnosis of Crohn's disease or symptoms indicative of CD (self or family members), and any exposure to raw milk, dairy cattle, and cattle with JD, either through employment or from living on a farm.

\section{Statistical Analysis}

The prevalence of CD was computed as the number of cases of CD divided by the total number of respondents overall and within exposure groups (producer exposed/ not exposed to Map, veterinarian exposed/not exposed to Map).

Descriptive statistics were generated for each exposure group. To determine whether associations existed between exposure to Map and CD, multivariable analyses were conducted using a matched case-control approach. Cases of CD were matched to randomly se- lected, noncase respondents, and matched 4:1 to each case by occupation (producer, veterinarian), state (Iowa, Michigan, Wisconsin), age category (10-yr age groups), and sex (male, female).

To quantitatively describe the association between exposure and risk factors on $\mathrm{CD}$, conditional logistic regression models for m:n matching (PROC PHREG, SAS 9.3.1) were developed for risk factors associated with exposure to Map (raw milk, dairy cattle, cattle with JD). Models were developed for each risk factor individually, and those found to be significantly associated with $\mathrm{CD}$ at $P \leq 0.05$ were considered for inclusion in multivariable analysis. A model including all potential risk factors was created and subjected to a backward model building approach to achieve the most parsimonious model, by repeatedly removing the risk factor with the highest $P$-value from the model until all risk factors were significant at $P \leq 0.05$. If removal of a risk factor resulted in changes in odds ratio of the major risk factor of interest (exposure to Map), the variable was considered a confounder and was maintained in the model. The final model was determined by the best combination of significance of risk factors retained in the model and model log-likelihood scores.

\section{RESULTS}

A total of 702 producer and 774 veterinarian surveys were successfully completed from 1,526 returned surveys (Table 1). The majority of producers surveyed were dairy producers (95\%), and $66 \%$ of producers ( $67 \%$ of dairy and $39 \%$ of beef) and $56 \%$ of veterinarians reported exposure to cattle infected with Map. The highest levels of exposure to cattle with JD were seen in respondents from Wisconsin $(71 \%)$, followed by Iowa (59\%), and Michigan (46\%). Dairy farmers had the highest exposure to cattle infected with JD (67\%), followed by veterinarians (56\%) and beef cattle farmers (39\%).

There were 7 cases of $\mathrm{CD}$ out of 1,476 study respondents, yielding a prevalence of 474.2 cases per 100,000 (Table 1). The highest prevalence of $\mathrm{CD}$ was seen in veterinarians (516.8 per 100,000), followed by dairy producers (448.4 per 100,000). The highest rates by state were seen in Iowa $(854.7$ per 100,000), followed by Wisconsin (407.7 per 100,000) and Michigan (395.3 per $100,000)$. The prevalence of CD in respondents exposed to $M a p$ was 446.9 per 100,000 (4 of 895 respondents) versus 516.4 per 100,000 in respondents with no reported exposure to Map (3 of 581). Study respondents also reported 41 cases of inflammatory bowel disease (1 beef, 9 dairy, and 31 veterinary cases).

Most dairy farmers (89.5\%) were raised on dairy farms, whereas $45.5 \%$ of beef farmers were raised on 
Table 1. Numbers of study participants with Crohn's disease, by state, sex, occupation, and potential exposure to Mycobacterium avium ssp. paratuberculosis $(\text { Map })^{1}$

\begin{tabular}{|c|c|c|c|c|c|c|c|c|}
\hline \multirow[b]{2}{*}{ Occupation } & \multirow[b]{2}{*}{ Sex } & \multirow[b]{2}{*}{ Map exposure } & \multicolumn{2}{|c|}{ Iowa } & \multicolumn{2}{|c|}{ Michigan } & \multicolumn{2}{|c|}{ Wisconsin } \\
\hline & & & $\mathrm{n}$ & $\mathrm{CD}$ & $\mathrm{n}$ & $\mathrm{CD}$ & $\mathrm{n}$ & $\mathrm{CD}$ \\
\hline \multirow{3}{*}{ Dairy producer } & & Negative & 1 & 0 & 9 & 0 & 8 & 0 \\
\hline & Male & Positive & 41 & 0 & 132 & 0 & 233 & 1 \\
\hline & & Negative & 11 & 1 & 94 & 0 & 95 & 0 \\
\hline \multirow{2}{*}{ Beef producer } & Male & Positive & 7 & 0 & 0 & 0 & 5 & 0 \\
\hline & & Negative & 6 & 0 & 0 & 0 & 11 & 0 \\
\hline \multirow[t]{4}{*}{ Veterinarian } & Female & Positive & 6 & 0 & 12 & 0 & 66 & 0 \\
\hline & & Negative & 21 & 0 & 41 & 1 & 52 & 1 \\
\hline & Male & Positive & 79 & 1 & 78 & 1 & 190 & 0 \\
\hline & & Negative & 56 & 0 & 129 & 0 & 44 & 0 \\
\hline
\end{tabular}

${ }^{1} \mathrm{n}=$ number of study participants; $\mathrm{CD}=$ number of cases of Crohn's disease.

beef or other types of farms, and $41 \%$ of veterinarians were raised on farms (Table 2). Potential exposure of respondents to Map through consumption of unpasteurized milk was common, with $94 \%$ of producers and $62 \%$ of veterinarians reporting this practice. Contact with cattle demonstrating clinical signs of JD (chronic, nonresponsive, intermittent diarrhea, progressive weight loss, normal appearance but unthrifty) was common: $59 \%$ of veterinarians, $56 \%$ of dairy producers, and $42 \%$ of beef producers reported contact with cattle with clinical signs of JD, and $59 \%$ of veterinarians, $71 \%$ of dairy producers, and $42 \%$ of beef producers reported contact with cattle with confirmed cases of JD.

The presence of family members with CD was assessed as an indicator of other possible exposures associated with CD (Table 2). Although 1 beef producer, 28 dairy producers, and 41 veterinarians reported having relatives with $\mathrm{CD}$, only 1 veterinarian with $\mathrm{CD}$ reported any family members with CD. Stress was reported in $45 \%$ of veterinarians, $43 \%$ of dairy producers, and

Table 2. Numbers of study participants with Crohn's disease with potential risk factors for Crohn's disease or exposure to Mycobacterium avium ssp. paratuberculosis, by type of respondent ${ }^{1}$

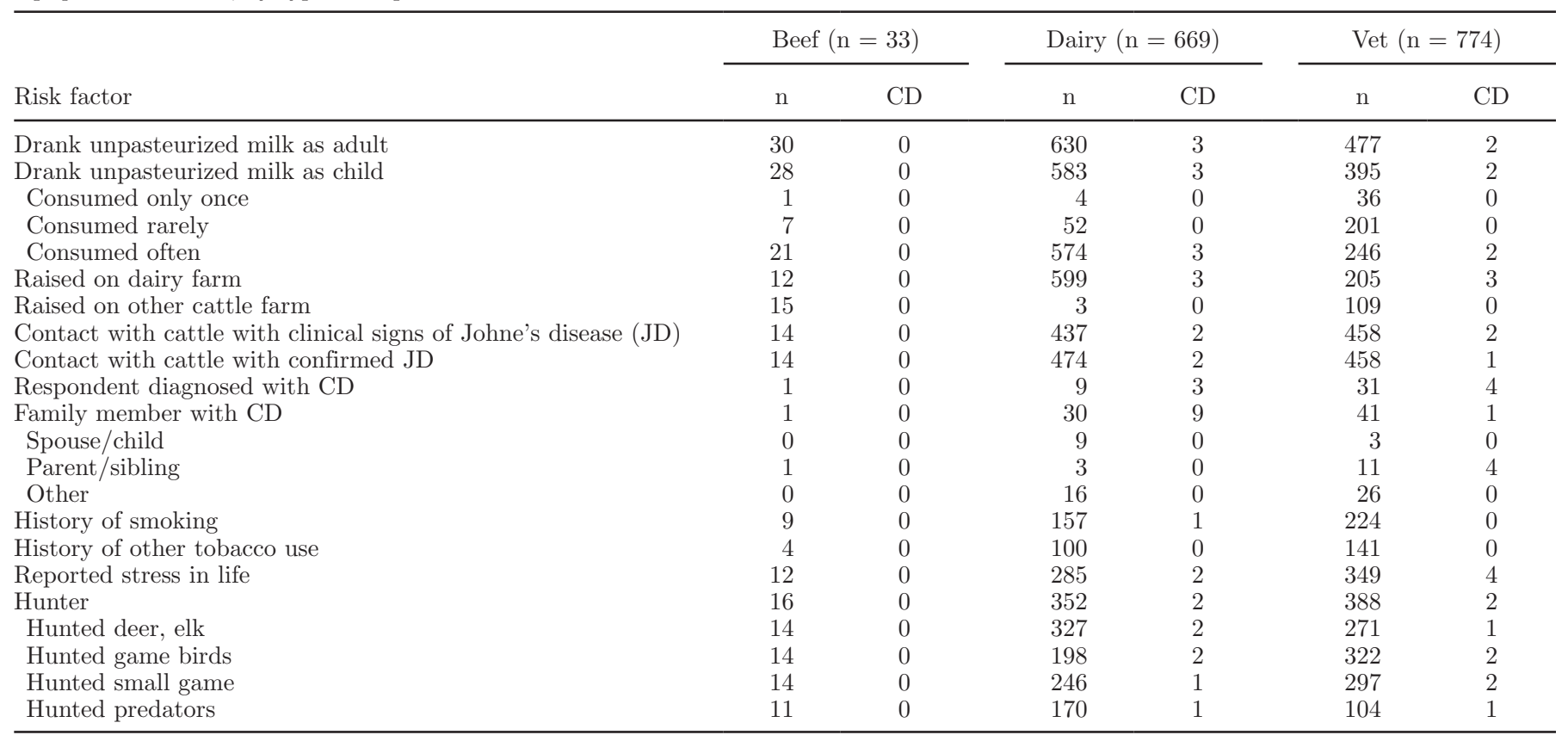

${ }^{1} \mathrm{n}=$ number of study participants; $\mathrm{CD}=$ number of cases of Crohn's disease. 
Table 3. Multivariable logistic regression for matched analysis for occurrence of Crohn's disease for 7 cases and 28 controls matched by respondent occupation, age, sex, and state

\begin{tabular}{|c|c|c|c|}
\hline Risk factor & $P$-value & Odds ratio & $95 \% \mathrm{CI}$ \\
\hline $\begin{array}{l}\text { Exposure to Map-infected cattle } \\
\text { Relative with Crohn's disease }\end{array}$ & $\begin{array}{l}0.3910 \\
0.2356\end{array}$ & $\begin{array}{l}2.5 \\
2.8\end{array}$ & $\begin{array}{l}0.3-20.7 \\
0.5-14.8\end{array}$ \\
\hline $\begin{array}{l}\text { Model log-likelihood }=17.36 \\
\text { Likelihood ratio } \chi^{2}=1.96,2 \text { df, } P=0.3756\end{array}$ & & & \\
\hline
\end{tabular}

${ }^{1}$ Map $=$ Mycobacterium avium ssp. paratuberculosis.

$36 \%$ of beef producers: all $4 \mathrm{CD}$ cases in veterinarians and 2 of $3 \mathrm{CD}$ cases in dairy producers reported stress. Cigarette smoking was not common $(<30 \%$ in all 3 respondent groups), and only 1 case of CD (dairy producer) reported a history of smoking. Hunting was reported by $53 \%$ of beef producers, $48 \%$ of dairy producers, and $50 \%$ of veterinarians, with 2 of $3 \mathrm{CD}$ cases in dairy producers and 2 of $4 \mathrm{CD}$ cases in veterinarians reporting hunting in the past. The majority of dairy producers and veterinarians were 40 to $49 \mathrm{yr}$ of age (Figure 1), whereas the majority of beef producers were 60 or older. No statistically significant associations were found between CD and any of the risk factors evaluated in this study at the univariable level.

A multivariable conditional logistic regression model was developed using a backward model building approach (Table 3), and the best regression model developed suggested that occupational exposure to cattle with JD and having a relative with $\mathrm{CD}$ tended to increase the odds of the respondent having the disease, but these associations were not statistically significant.

\section{DISCUSSION}

The prevalence of CD in this study (474 per 100,000) was higher than that reported in a survey of dairy producers with JD in the United Kingdom (128.7 per 100,000; Jones et al., 2006) and in kibbutz dwellers in Israel (65.1 per 100,000; Niv et al., 1999), but agreed with these studies in finding no association between the occurrence of $\mathrm{CD}$ and exposure to Map through cattle with JD. In one study (Jones et al., 2006), the prevalence of $\mathrm{CD}$ in dairy producers was not different from the prevalence in the general population, whereas the study conducted in Israel found a lower prevalence of $\mathrm{CD}$ in residents of the agricultural setting of the kibbutz compared with the rest of the country (Niv et al., 1999).

There were no statistically significant associations observed between exposure to cattle herds with JD and occurrence of $\mathrm{CD}$ in producers or veterinarians in this study, which may be because of the low number of cases of CD reported. The use of the matched case-control study approach was intended to provide additional power to the study, but it yielded no significant associations between risk factors and the occurrence of CD.

An association between CD and Map was not demonstrated in this study, and current research on the topic has been divided. Much of the argument for a causal association between Map and CD has been based on finding viable $\mathrm{Map}$ in intestinal lesions (McFadden et al., 1987; Bull et al., 2003; Autschbach et al., 2005; Sechi et al., 2005) and peripheral blood (Naser et al., 2004) of $\mathrm{CD}$ patients. One meta-analysis of 28 case-control studies of Map in CD patients found the odds of finding $M a p$ in tissue samples from CD patients to be 7.0 using PCR and 1.7 using ELISA to detect antibodies in serum (Feller et al., 2007). However, some studies have found Map in patients who did not have CD (Sechi et al., 2005; Feller et al., 2007), and other studies have found no Map in tissues or peripheral blood of patients with CD (Freeman and Noble, 2005; Lozano-Leon et al., 2006). Differences in these studies may be attributable to different biases within each study and to differences in Map culture methods (Naser and Collins, 2005). Although finding viable Map in CD patients suggests an association between the two, the temporal association

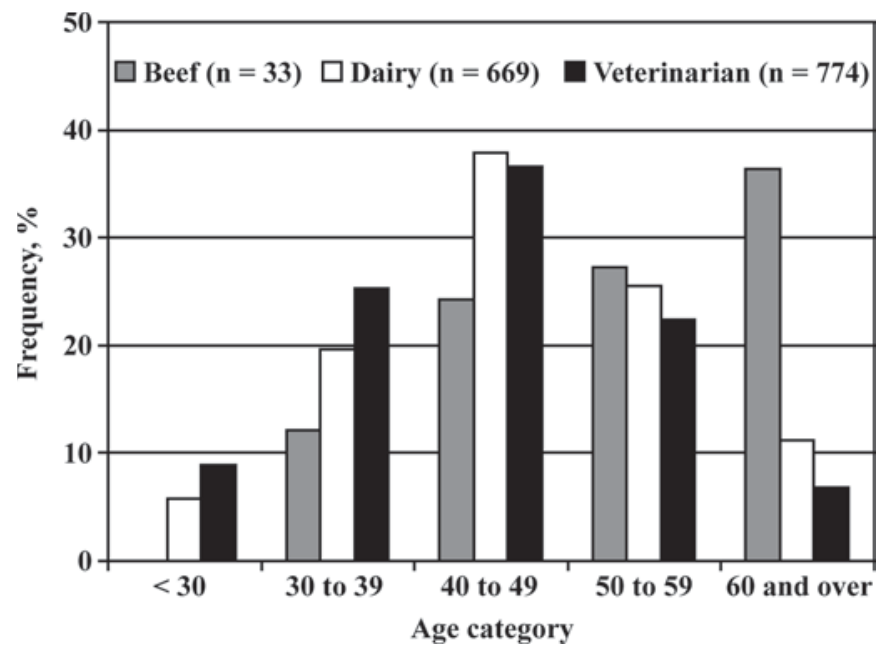

Figure 1. Age of respondents in years, by type of respondent. 
between the presence of Map and later development of CD has been difficult to demonstrate conclusively (Sechi et al., 2005). Some researchers believe that the temporality of the association has been demonstrated, based on findings that show that Map harvested from CD patients can result in disease in goats (Uzoigwe et al., 2007), but comparable findings in humans have not been found. Recently, it has been suggested in several studies that Map itself may not be sufficient to cause $\mathrm{CD}$, but that certain host mutations, including NOD2/ CARD15 (nucleotide-binding oligomerization domain containing 2 or caspase recruitment domain family, member 15), serve a role in bacterial sensing and activation of innate immune responses, providing a possible link between $\mathrm{CD}$ and a bacterial trigger such as Map (Behr and Schurr, 2006). Others have concluded that the causal association is not clear but that Mapassociated CD needs to be acknowledged even though the etiology of the process is not understood (Selby, 2004; Chamberlin et al., 2007).

Given conflicting evidence from studies based on bacterial detection in patients with $\mathrm{CD}$, studies examining the association between exposure to Map and CD can provide a different insight into the association between Map and CD. One study in Israel found no difference in rates of CD between kibbutz residents and the rest of the country (Niv et al., 1999), which suggests that contact with livestock was not associated with increased CD risk. In a cross-sectional study of dairy farmers in the United Kingdom (Jones et al., 2006), no association was found between exposure to clinical cases of JD and the development of $\mathrm{CD}$, but associations were found between ulcerative colitis and age, frequency of contact with cattle, and smoking. A UK case-control study of $\mathrm{CD}$ with exposure to drinking water and dairy products (potential sources of Map) with CD showed no significant association with measures of potential contamination of water sources with Map, water intake, or water treatment and CD (Abubakar et al., 2007). However, the same study found that consumption of pasteurized milk was associated with reduced risk of CD, indicating that dairy products potentially contaminated with Map were not important factors in the etiology of CD. Overall, these studies indicate no clear association between exposure to Map or JD and the development of CD.

\section{CONCLUSIONS}

There were no statistically significant associations observed between exposure to cattle with Johne's disease and Crohn's disease in producers and veterinarians. Despite the use of a cross-sectional survey to overcome the limitations of the low number of cases reported herein, there were insufficient cases for adequate power using this study design. Although the current study does not offer conclusive proof of a lack of association between Map and Crohn's disease, it adds to the body of evidence that, if such an association exists, there are probably other more important risk factors that are likely to be directly associated with the development of Crohn's disease.

\section{ACKNOWLEDGMENT}

This study was supported by grants from the Iowa Livestock Health Advisory Council and US Department of Agriculture.

\section{REFERENCES}

Abubakar, I., D. J. Myhill, A. R. Hart, I. R. Lake, I. Harvey, J. M. Rhodes, R. Robinson, A. J. Lobo, C. S. Probert, and P. R. Hunter. 2007. A case-control study of drinking water and dairy products in Crohn's disease - Further investigation of the possible role of Mycobacterium avium paratuberculosis. Am. J. Epidemiol. 165:776-783.

Autschbach, F., S. Eisold, U. Hinz, S. Zinser, M. Linnebacher, T. Giese, T. Loffler, M. W. Buchler, and J. Schmidt. 2005. High prevalence of Mycobacterium avium subspecies paratuberculosis IS900 DNA in gut tissues from individuals with Crohn's disease. Gut 54:944-949.

Behr, M. A., and E. Schurr. 2006. Mycobacteria in Crohn's disease: A persistent hypothesis. Inflamm. Bowel Dis. 12:1000-1004.

Bull, T. J., E. J. McMill, K. Sidi-Boumedine, A. Skull, and D. Durkin. 2003. Detection and verification of Mycobacterium avium ssp. paratuberculosis in fresh ileocolonic mucosal biopsy specimens from individuals with and without Crohn's disease. J. Clin. Microbiol. 41:2915-2923.

Chamberlin, W., T. Borody, and S. Naser. 2007. MAP-associated Crohn's disease: MAP, Koch's postulates, causality, and Crohn's disease. Dig. Liver Dis. 39:792-794.

Chiodini, R. J. 1989. Crohn's disease and mycobacterioses; A review and comparison of two disease entities. Clin. Microbiol. Rev. 1:90-117.

Ellingson, J. L., J. L. Anderson, J. J. Koziczkowski, R. P. Radcliff, S. J. Sloan, S. E. Allen, and N. M. Sullivan. 2005. Detection of viable Mycobacterium avium ssp. paratuberculosis in retail pasteurized whole milk by two culture methods and PCR. J. Food Prot. 68:966-972.

Feller, M., K. Huwiler, R. Stephan, E. Altpeter, A. Shang, H. Furrer, G. E. Pfyffer, M. W. Buchler, T. Jemmi, A. Baumgartner, and M. Egger. 2007. Mycobacterium avium subspecies paratuberculosis and Crohn's disease: A systematic review and meta-analysis. Lancet Infect. Dis. 7:607-613.

Freeman, H. J., and M. Noble. 2005. Lack of evidence for Mycobacterium avium subspecies paratuberculosis in Crohn's disease. Inflamm. Bowel Dis. 11:782-783.

Jones, P. H., T. B. Farver, B. Beaman, B. Cetinkaya, and K. L. Morgan. 2006. Crohn's disease in people exposed to clinical cases of bovine paratuberculosis. Epidemiol. Infect. 134:49-56.

Lisby, G., J. Anderson, K. Engbaek, and V. Binder. 1994. Mycobacterium paratuberculosis in intestinal tissue from patients with Crohn's disease demonstrated by nested primer polymerase chain reaction. Scand. J. Gastroenterol. 29:923-929.

Lozano-Leon, A., M. Barreiro-de Acosta, and J. E. Dominguez-Munoz. 2006. Absence of Mycobacterium avium subspecies paratuberculosis in Crohn's disease Patients. Inflamm. Bowel Dis. 12:1190-1192.

McFadden, J. J., P. D. Butcher, R. Chiodini, and J. Hermon-Taylor. 1987. Crohn's Disease-Isolated Mycobacteria are identical to Mycobacterium paratuberculosis, as determined by DNA probes 
that distinguish between mycobacterial species. J. Clin. Microbiol. 25:796-801.

Naser, S. A., and M. T. Collins. 2005. Debate on the lack of evidence of Mycobacterium avium ssp. paratuberculosis in Crohn's disease. Inflamm. Bowel Dis. 11:1123.

Naser, S. A., G. Ghobrial, C. Romero, and J. F. Valentine. 2004 Culture of Mycobacterium avium subspecies paratuberculosis from the blood of patients with Crohn's disease. Lancet 364:10391044.

Niv, Y., G. Abuskis, and G. M. Fraser. 1999. Epidemiology of Crohn's disease in Israel: A survey of Israeli kibbutz settlements. Am. J. Gastroenterol. 94:2961-2965.

Pickup, R. W., G. Rhodes, S. Arnott, K. Sidi-Boumedine, T. J. Bull, A. Weightman, M. Hurley, and J. Herman-Taylor. 2005. Mycobacterium avium ssp. paratuberculosis in the catchment area and water of the River Taff in South Wales, United Kingdom, and its potential relationship to clustering of Crohn's disease cases in the City of Cardiff. Appl. Environ. Microbiol. 71:2130-2139.

Sechi, L. A., A. M. Scanu, P. Molicotti, S. Cannos, M. Mura, G. Dettori, G. Fudda, and S. Zanetti. 2005. Detection and isolation of Mycobacterium avium subspecies paratuberculosis from intestinal mucosal biopsies of patients with and without Crohn's disease in Sardinia. Am. J. Gastroenterol. 100:1529-1536.

Selby, W. S. 2004. Mycobacterium avium subspecies paratuberculosis bacteremia in patients with inflammatory bowel disease. Lancet 364:1013-1014.

Uzoigwe, J. C., M. L. Khaitsa, and P. S. Gibbs. 2007. Epidemiological evidence for Mycobacterium avium subspecies paratuberculosis as a cause of Crohn's disease. Epidemiol. Infect. 135:1057-1068.

Van Kruiningen, H. J. 1999. Lack of support for a common etiology in Johne's disease of animals and Crohn's disease in humans. Inflamm. Bowel Dis. 5:183-191. 